\title{
A narrative review of interstitial lung disease in anti-synthetase syndrome: a clinical approach
}

\author{
Naina Sawal $^{1}$, Sanjay Mukhopadhyay ${ }^{2}$, Sheetal Rayancha ${ }^{3}$, Alastair Moore ${ }^{4}$, Puneet Garcha ${ }^{5}$, \\ Anupam Kumar ${ }^{5}$, Viren Kaul ${ }^{6 \wedge}$ \\ ${ }^{1}$ Department of Internal Medicine, SUNY Upstate Medical University, Syracuse, NY, USA; ${ }^{2}$ Department of Pathology, Cleveland Clinic, Cleveland, \\ OH, USA; ${ }^{3}$ Department of Rheumatology, SUNY Upstate Medical University, Syracuse, NY, USA; ${ }^{4}$ Department of Radiology, Baylor Scott and \\ White Health, Dallas, TX, USA; ${ }^{5}$ Department of Pulmonary Critical-Care, Baylor College of Medicine, Houston, TX, USA; ${ }^{6}$ Department of \\ Pulmonary Critical-Care, Crouse Health/SUNY Upstate Medical University, Syracuse, NY, USA \\ Contributions: (I) Conception and design: All authors; (II) Administrative support: N Sawal; (III) Provision of study materials or patients: All authors; \\ (IV) Collection and assembly of data: All authors; (V) Data analysis and interpretation: All authors; (VI) Manuscript writing: All authors; (VII) Final \\ approval of manuscript: All authors. \\ Correspondence to: Naina Sawal. Department of Internal Medicine, SUNY Upstate Medical University, 750 E Adams Street, Syracuse, NY 13210, \\ USA. Email: sawal.naina@mayo.edu.
}

\begin{abstract}
Anti-synthetase syndrome (AS) is a rare autoimmune disorder characterized by the presence of aminoacyl-transfer RNA synthetase antibodies in conjunction with clinical features such as interstitial lung disease (ILD), Raynaud's phenomenon, nonerosive arthritis, and myopathy. AS distinguishes itself from other inflammatory myopathies by its significant lung involvement and rapidly progressive interstitial lung disease (AS-ILD), therefore the management of AS-ILD requires careful clinical, serologic and radiologic assessment. Glucocorticoids are considered the mainstay of therapy; however, additional immunosuppressive agents are often required to achieve disease control. Patient prognosis is highly dependent on early diagnosis and symptom recognition as the antibody profile is thought to influence therapy response. Since progressive ILD is the leading cause of morbidity and mortality, this review will discuss the clinical approach to patient with suspected AS, with particular emphasis on diagnosis and management of AS-ILD.
\end{abstract}

Keywords: Antisynthetase syndrome; interstitial lung disease; computed tomography; immunosuppressive agents; autoimmune disease

Submitted Nov 18, 2020. Accepted for publication Jul 23, 2021.

doi: $10.21037 /$ jtd-20-3328

View this article at: https://dx.doi.org/10.21037/jtd-20-3328

\section{Introduction}

Idiopathic inflammatory myopathies (IIM) include a heterogenous class of autoimmune disorders characterized by their shared symptoms of muscle inflammation (1). Polymyositis (PM), dermatomyositis (DM), inclusion body myositis (IBM), and necrotizing autoimmune myopathy are the four major groups within this category (2). While PM and DM were initially thought to be independent diseases, recent research has identified the presence of additional antibodies in a subset of these patients. Whereas many of their symptoms were phenotypically similar to PM and DM, studies found that ILD frequently predominated at presentation (3) and contributed to the high morbidity and mortality rates in these patients. Although the presence of ILD in PMDM has been reported to range between 5-30\% (4), a study of 203 patients with these specific antibodies found the prevalence of ILD to be as high as $86 \%$ (3).

^ ORCID: Naina Sawal, 0000-0001-9317-7179; Viren Kaul, 0000-0003-3047-4840. 
Due to the different rates of ILD acuity, progression, and lung involvement in this specific patient population this phenomenon has been recognized as a distinct entity outside of PM and DM, termed anti-synthetase syndrome (AS). While it has managed to gain some recognition over the past few years, the lack of both physician awareness and large-scale research projects, results in it being an under-diagnosed cause of interstitial lung disease (ILD). We present the following article in accordance with the Narrative Review reporting checklist (available at https:// dx.doi.org/10.21037/jtd-20-3328).

\section{Methods}

Incidence data was gathered from a variety of databases including: JSTOR, PubMed database, Google scholar (http://www.scholar.google.com) and Cochrane library (http://www.cochranelibrary.com). The keywords specified to search for the articles include: ILD, AS, polymyositis, dermatomyositis, tRNA-synthetase antibody, lung transplant, cryobiopsy, retrospective, prospective, and meta-analysis. In order to ensure the information presented is valid and applicable to the topic, almost all of the articles chosen were be published between 2001 and 2019. Additionally, due to the lack of consensus on research areas on this topic, the study populations selected included both male and female patients of any age group and in any geographic setting with ILD. Lastly, in order to avoid any misunderstandings in discourse, only papers published in English were selected for and non-human studies were discarded. In conjunction with gathering data from the publications described, physician input was also obtained from respected pulmonologists, rheumatologists, radiologists, and pathologists in the field in order to create, what we hope is a succinct review of the diagnosis and management of this condition.

\section{Definition}

AS is characterized by antibodies directed against an aminoacyl-transfer RNA (tRNA) synthetase along with a constellation of clinical findings/symptoms including "mechanic's hands", ILD, myositis, nonerosive arthritis and Raynaud's phenomenon (3). However, it is key to note that patients are not required to have all of the manifestations of the disease in order to ascertain a diagnosis. The syndrome is considered to be present in patients with an anti-synthetase antibody along with two of the following features: ILD, inflammatory myopathy, or inflammatory polyarthritis (5).

Historically, AS was considered to be a clinical subgroup within the DM/PM cohort, due to overlapping clinical features and antibody profile. Research suggests that patients with AS have a higher prevalence of ILD (6), that is rapidly progressive, as compared to those with DM or PM (7). Therefore, due to its clinical trajectory, response to treatment and prognosis that AS should be viewed as a distinct, stand-alone phenotype.

\section{History and epidemiology}

In 1981, Walker et al. discovered anti-Jo-1, an aminoacyltRNA synthetase antibody frequently occurring in a cohort of their patients with DM and PM (8). In 1984, Bernstein et al. found histidyl-tRNA synthetase to be the target of the Jo-1 autoantibodies (9) and by the early 1990s patients with anti-Jo-1 antibodies were recognized as having a distinct phenotype now termed "AS". Since the discovery of Jo1 , ten additional tRNA synthetase antibodies have been identified including: anti-PL-7 (threonyl), anti-PL-12 (alanyl), anti-EJ (glycyl), anti-KS (asparaginyl), anti-OJ (isoleucyl), anti-YRS/Ha (tyrosyl), anti-SC (lysyl), anti-Zo (phenylalanyl), anti-JS (glutaminyl) and anti-Wa (directed against NEFA, a tRNA related protein), with all of these less commonly encountered than Jo-1 (10,11).

Due to the rare nature of the disease, and likely underidentification, the literature has been limited to case series and reports. The disease generally predominantly affects women (with a mean female/male ratio 2:1) (12). Comprehensive data collection is also made difficult by lack of clinician awareness, with patients often misdiagnosed as having either idiopathic pulmonary fibrosis, DM or PM $(13,14)$. As a result, the prevalence and the annual incidence of the disease remain largely unknown. The annual incidence of $\mathrm{PM} / \mathrm{DM}$ is $2-10$ new cases per million persons and roughly 30 percent of patients have been found to have a clinical presentation that may be consistent with AS (15).

\section{Diagnostic criteria}

Although many scientific models have been proposed to aid in the diagnosis of AS, we will be focusing on the criteria outlined by Connors and Solomon as they are the most reviewed (Table 1). The first, proposed by Connors et al. (16) recommends that all patients with AS must have evidence of a tRNA synthetase autoantibody, in addition to one or 
Table 1 Proposed diagnostic criteria for AS

\author{
Solomon et al. (2011) \\ Required: presence of anti-aminoacyl tRNA synthetase antibody \\ Plus: two major criteria or one major and two minor criteria: \\ Major: \\ (I) Interstitial lung disease (not attributable to another cause) \\ (II) Polymyositis or dermatomyositis (by Bohan and Peter criteria) \\ Minor: \\ (I) Arthritis \\ (II) Raynaud's phenomenon \\ Connors et al. (2010) \\ Required: presence of anti-aminoacyl tRNA synthetase antibody \\ Plus: one or more of the following clinical features: \\ (I) Raynaud's phenomenon \\ (II) Arthritis \\ (III) Interstitial lung disease \\ (IV) Fever (not attributable to another cause) \\ (V) Mechanic's hands
}

(III) Mechanic's hands (thickened and cracked skin on hands, particularly at fingertips)

AS, anti-synthetase syndrome.

more clinical features including: constitutional symptoms (e.g., fever, weight loss), "mechanic's hands", ILD, myositis, nonerosive arthritis, and Raynaud's phenomenon (16). A separate set of criteria was introduced in 2011 by Solomon et al. (12), who considered major clinical criteria to include ILD and fulfillment of Bohan and Peter (17) criteria for DM/PM. The proposed minor criteria were arthritis, Raynaud's phenomenon, and mechanic's hands. For diagnosis of AS, patients need to have two major or one major and two minor criteria in addition to the presence of an aminoacyl-tRNA synthetase (Table 1). Although data is limited, one observational study found that patients who met Solomon's criteria at disease onset presented with incomplete clinical forms and clinical progression favored the eventual fulfillment of Connor's criteria (18). Their results suggested that while Connor's criteria can be considered in the initial consideration for AS, Solomon's criteria can be considered the gold standard for diagnosis. Since patients often present on a spectrum, physicians are more likely to make the diagnosis of AS based on Solomon's criteria as the presence of mechanic's hands or ILD are both highly associated with classic presentation of AS.
A study of 828 patients with diagnosed AS found that while the triad findings were similar amongst the cohort groups, the onset mainly began with a single triad finding in all groups (19). Similarly, Cavagna et al. also found that most anti-Jo-1 anti-synthetase patients can present with the incomplete clinical form at disease onset and often manifest the full triad during the course of the disease (19). Thus, in patients presenting with only one clinical feature and there is clinical suspicion of AS, we recommend utilizing Connor's criteria as an initial diagnostic test since it is more likely to capture the diagnosis of AS. Consequently, Solomon's criteria can then serve as a confirmatory test for high-risk patients positive to anti-synthetase antibodies (18).

\section{Clinical features}

The six hallmark symptoms of AS are: fever, myositis, ILD, inflammatory polyarthritis, Raynaud's phenomenon and "mechanic's hands" (thickened skin of tips and margins of fingers) (Figure 1) (20). While all AS patients may share similar clinical features, research suggests that the heterogeneous presentation of the disease may be due to the 

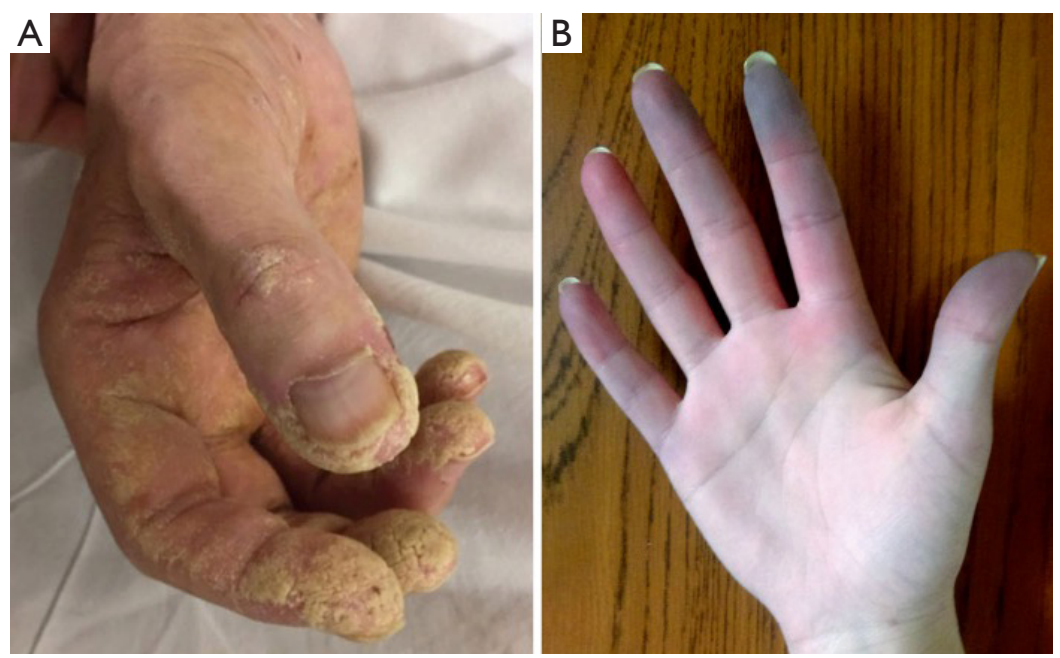

Figure 1 Clinical features of two patients with AS. (A) A patient with anti-Jo-1 positive AS and hyperkeratosis visible on his hand ("mechanic hands"). (B) A patient with Raynaud's phenomenon. Image use with permission from Wernham M, Montague S. Mechanic's hands and hiker's feet in antisynthetase syndrome. Reproduced by permission of CMAJ Group. Figure 1A from Ref. (20). AS, anti-synthetase syndrome.

Table 2 Known antibodies targeted against antigens

\begin{tabular}{lc}
\hline Anti-ARS antibody & Antigen \\
\hline Anti-Jo-1 & Histidyl \\
Anti-PL7 & Theronyl \\
Anti-PL12 & Alanyl \\
Anti-OJ & Isoleucyl \\
Anti-EJ & Glycyl \\
Anti-KS* & Asparaginyl \\
Anti-Zo* & Phenylalanyl \\
Anti-SC* & Lysyl \\
Anti-JS* & Glutaminyl \\
Anti-YRS/Ha* & Tyrosyl \\
Anti-Wa* & NEFA* \\
\hline
\end{tabular}

${ }^{*}$, lab test commercially unavailable.

different proportions of specific antibodies found in each patient $(21,22)$.

Hamaguchi et al. compared the demographic features of 165 Japanese patients with AS and found no differences in age of onset or sex regardless of antibody subgroup. Additionally, they compared muscle weakness and ILD among individual antibody subgroups, both at the initial visit and during the entire follow-up period. While patients with anti-Jo-1, anti-EJ, and anti-PL-7 had myositis progression, those with anti-PL-12, anti-KS, and antiOJ demonstrated relatively myopathy. In contrast, almost all patients eventually developed ILD (23) and those with ILD during their initial visit had disease progression. In Trallero-Araguas et al., a multi-center study that included 148 anti-Jo-1 patients, they found that most patients (77.2\%) had isolated lung, muscle or joint involvement at disease onset (24). Because these symptoms may occur individually or in a variety of combinations, establishing a diagnosis can be challenging. The development of ILD may precede (10$30 \%$ ), occur concurrently $(53-70 \%)$ or follow $(6-20 \%)$ the onset of myositis (25). It is this lack of delineation among symptom presentation that often results in misdiagnosis and delayed initiation of treatment.

\section{Autoantibodies and AS-ILD}

Aminoacyl-tRNA synthetase is an enzyme that attaches a specific amino acid to a corresponding tRNA (Table 2). Anti-synthetase antibodies, target and attack tRNA synthetase thereby prompting recruitment of antigenpresenting and inflammatory cells to the site of muscle or lung injury (13). Of the eleven known antibodies, antiJo1 anti-histidyl tRNA synthetase is the most commonly detected, with studies reporting a range from $60-80 \%(26,27)$. Other anti-synthetase antibodies are far less common, with anti-PL7/anti-PL12 reported in $2-5 \%$ of patients and the remaining 8 antibodies in $<2 \%$ of patients (28). Although the 
exact pathogenesis behind these anti-synthetase antibodies is unclear, one hypothesis suggests that they are produced after a "trigger" event like a viral infection or drug exposure (29).

While the diagnostic criteria proposed by both Connors and Solomon mandate the presence of an anti-synthetase antibody, the absence of an antibody does not preclude the diagnosis since autoantibody levels can fluctuate depending on disease activity and treatment $(9,30,31)$. Similarly, of the twenty existing tRNA synthetases only eleven autoantibodies have been discovered, so lack of autoantibody detection should not be used to definitively rule out AS. Additionally, laboratory testing strategies can also influence results. Immunoprecipitation is considered to be the gold standard for diagnosis however ELISA testing has greater sensitivity at detecting low anti-Jo1 titers compared to double immunodiffusion (DID) (32). A retrospective analysis from Japan highlighted these differences when they evaluated 64 patients with idiopathic inflammatory muscle disease and found ELISA testing identified an additional 13 patients with weakly positive anti-Jo-1 activities thought to be due to fluctuating titer levels (33).

Further, it is well recognized that patients initially diagnosed with "idiopathic" interstitial pneumonia may ultimately be found to have AS through the discovery of additional anti-synthetase antibodies (14,34). Fischer et al. examined 37 patients who despite having clinical features suggestive of AS were diagnosed with "idiopathic" interstitial pneumonia based on negative anti-Jo-1 serology (34). After testing for the presence of other anti-synthetase antibodies, nine were confirmed to have either anti-PL-7 and antiPL-12 and eventually diagnosed with non-anti-Jo-1 positive AS (34). Watanabe et al. conducted a similar retrospective analysis of 198 patients to elucidate the prevalence of the anti-synthetase positive subpopulation among idiopathic interstitial pneumonias. Through additional testing, they found 13 of those patients to be positive for anti-synthetase antibodies (anti-EJ and anti-PL12) (14).

Recent evidence suggests that both the clinical picture and outcome of AS are intimately tied to the anti-synthetase antibody being expressed $(26,35,36)$. Two separate retrospective analyses involving 133 and 233 anti-synthetase patients, respectively, found that anti-Jo-1 antibody was associated with a more diffuse phenotype while patients with anti-PL-7, anti-PL-12, anti-Ks and anti-OJ sole manifestation was ILD $(3,37)$. Many other studies, including Marie et al. compared the characteristics between antisynthetase antibody patients with anti-Jo-1 and anti-PL7/ PL12, and concluded that while the presence of anti-Jo-1 was associated with more severe myositis, both anti-PL7/ PL12 was associated with early and severe ILD $(20,38,39)$.

This concept of phenotypic heterogeneity has important implications in both symptom presentation, early diagnosis and overall prognosis (38) since ILD is the main mortality determinant in AS. Trallero-Araguás et al. demonstrated that most anti-Jo-1 patients develop ILD which stabilizes with immunosuppressive treatment (24). Likewise, Aggarwal et al. conducted a prospective analysis of 202 patients and found non-Jo-1 positive patients have decreased survival compared to anti-Jo-1 positive patients with a 5-and 10 -year unadjusted survival of $90 \%$ and $70 \%$ in anti-Jo-1 positive patients, and $75 \%$ and $47 \%$ in non-Jo-1 positive patients (35). The presence of anti-PL-12 antibodies was reported to constitute a subset of patients who may have a more favorable prognosis compared to anti-Jo-1 positive patients (39). Unfortunately, it is difficult to extrapolate the causation behind the decreased survival as patients with non-Jo-1 anti-synthetase autoantibodies may also experience delays in diagnosis since these antibodies are not widely included in all commercially available clinical assays.

\section{HCRT findings in AS-ILD}

As with other forms of ILD, high resolution CT scans (HRCT) is the gold standard thoracic imaging modality. The most common radiological patterns noted in patients with AS on HCRT are non-specific interstitial pneumonia (NSIP), organizing pneumonia (OP), or mixed NSIPOP (40) ranging up to $55.6 \%, 21 \%$ and $24 \%(41,42)$, respectively. NSIP is characterized by extensive groundglass opacities, micronodules, discrete cysts, significant mosaic perfusion and air trapping, and consolidation. HRCT features of NSIP combined with subpleural and peribronchovascular areas of consolidation, resembling OP, seem to be relatively common in AS (43). These abnormalities, as seen in Figure 2, are limited to the extremes of the lung bases, essentially hugging or "pancaking" the diaphragm, which is a feature associated with AS (34).

OP is characterized by patchy, peripheral, often frankly subpleural, and peribronchiolar consolidation that may migrate. Combined NSIP-OP is characterized by a basal-predominant fibrotic abnormality which shows superimposed OP (34). Since NSIP and OP are often associated with active inflammation, it is important to note that these consolidations often decrease or disappear in $>90 \%$ of patients after the initiation of treatment (42). 

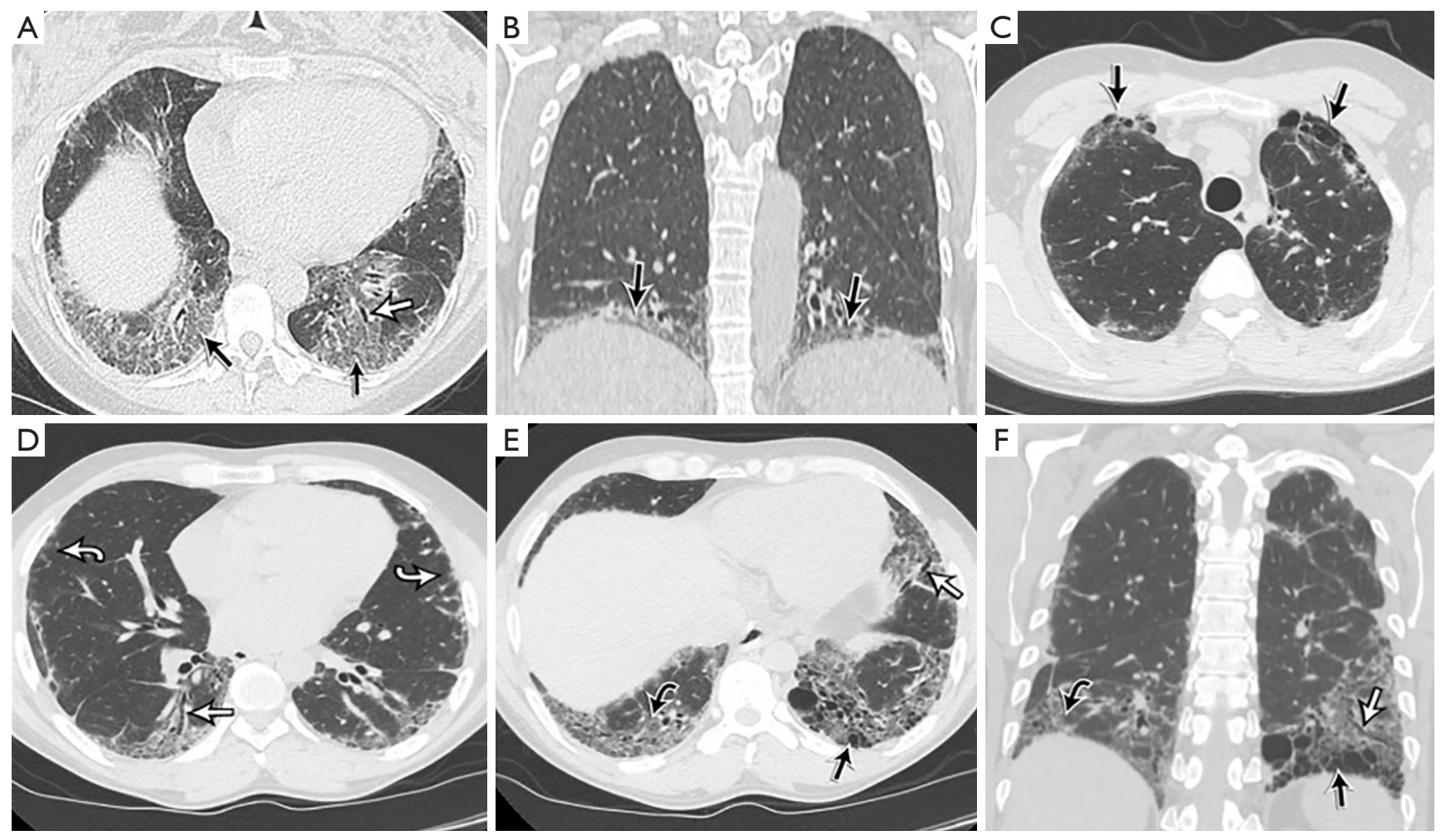

Figure 2 A summary of the most common radiological findings found in AS. (A,B) A woman in the 4th decade of her life with respiratory symptoms, dyspnea, rash, joint pain, sicca symptoms, and Raynaud's phenomenon with anti-EJ antibodies. HRCT images with an axial reconstruction through the lung bases (A) and a coronal reconstruction (B) show basal ground glass opacities (black arrow) associated with focal volume loss and traction bronchiectasis (white arrow). The opacities are consistent with NSIP; note that the abnormalities are limited to the extremes of the lung bases, essentially hugging or "pancaking" the diaphragm, which is a feature associated with AS. (C-F) A man in the fifth decade of his life presenting with cough and autoimmune workup demonstrating anti-Jo-1 antibodies. The patient improved significantly following treatment with prednisone and MMF. HRCT images with axial (C-E) and coronal (F) reconstructions show fibrosis involving the anterior upper lobes and both lung bases, manifested by subpleural and basal honeycomb cyst formation (straight black arrow), traction bronchiectasis (straight white arrow), mild ground glass opacity (curved black arrow) and irregular reticulation (curved white arrow). The presence of anterior upper lobe honeycombing in addition to lower lobe honeycombing (the latter is often seen with UIP) is a less common sign associated with CTD-associated ILD, which is normally associated with a typical NSIP pattern. AS, anti-synthetase syndrome; ILD, interstitial lung disease.

However, those with isolated OP have a better prognosis than those with either diffuse alveolar lesions or interstitial pneumonia (44).

Usual interstitial pneumonia (UIP) can also be seen and is characterized by honeycombing and traction bronchiectasis. Due to the lack of effective therapeutic options, disease progression often presents as honeycombing with associated fibrosis (41). The groundglass opacities and traction bronchiectasis may improve or remain unchanged with treatment (42). The presence of anterior upper lobe honeycombing in addition to lower lobe honeycombing (often seen with UIP) is a less common sign associated with CTD-associated ILD, which is normally associated with a typical NSIP pattern (45). Common CT findings at initial diagnosis include groundglass opacities, reticulations, and traction bronchiectasis in the lower lobes (42). A study of 64 patients also found CT findings predominantly in the lower lobe in addition to peripheral opacities and peribronchovascular lesions in similar distribution patterns (41). Other CT findings included lower volume loss, interlobular septal thickening and thickening of bronchovascular bundles.

High resolution computed tomography (HRCT) is another key component in the multidisciplinary approach to the diagnosis and management of ILD. Richards et al. conducted a cohort study assessing the clinical features of 

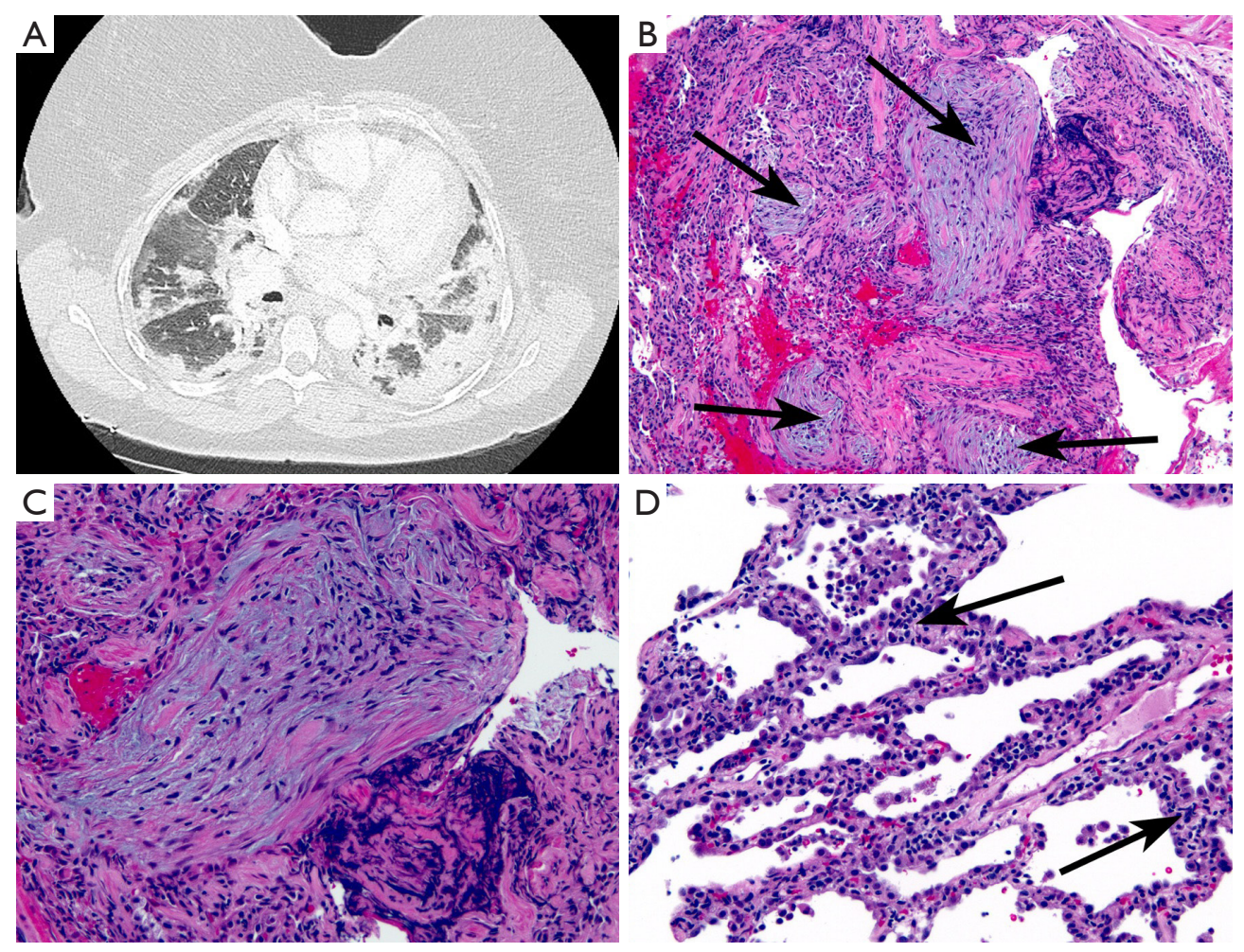

Figure 3 Transbronchial biopsy from a woman in 7th decade of life with AS, positive for anti-Jo-1. (A) Chest CT showed bilateral peripheral and lower lobe predominant consolidative and ground-glass opacities interpreted as mixed OP/NSIP. (B) Transbronchial biopsy showing organizing pneumonia (arrows indicate fibroblast plugs; HE, $\times 100$ ). (C) Fibroblast plug (Masson body) at higher magnification. (D) Diffuse interstitial chronic inflammation (arrows; HE, $\times 200$ ). These findings could be interpreted as a combination of organizing pneumonia and NSIP (HE, $\times 200$ ).

patients with anti-Jo-1 antibody. Several CT scans showed abnormalities in patients with no overt clinical symptoms of dyspnea or cough, highlighting the sensitivity of HRCT in detecting subclinical ILD (even in cases with normal PFTs) (46). In this respect, serial HRCT examinations may provide valuable information which supplement clinical history and other diagnostic tests, including pulmonary function tests. Important roles of HRCT in this context include assisting in determining prognosis, monitoring for the efficacy of treatment, and detecting progression of disease.

A multidisciplinary discussion (MDD) is often helpful in establishing a diagnosis of ILDs and this is especially important with AS. Given the diagnostic challenge of AS, MDD helps establish a diagnosis, in part by institution of early additional investigations in addition to implementation of a multidisciplinary treatment and follow up plan. The multidisciplinary teams typically consist of ILD-focused pulmonologists, chest radiologist, pathologist \& ideally a rheumatologist with ILD expertise $(47,48)$.

\section{Histopathologic features of AS}

Lung biopsy, including transbronchial biopsy, video-assisted thoracoscopic surgical biopsy (VATS) and surgical open lung biopsy (SLB) are infrequently utilized in patients with AS since (as currently defined) the diagnosis rests on a combination of physical examination, serology and HRCT findings there is limited role for bronchoscopy other than to rule out infections. Attempts to obtain biopsy are often precluded by severity of illness as well as these patients often present with rapidly progressive ILD and significant pulmonary function impairment. Transbronchial biopsy is of limited value due to the suboptimal amount of tissue obtained-the presence of areas of OP may not fully describe the pathology of affected lung as a whole (Figure 3). Cryobiopsy has been proposed as a less invasive substitute to surgical lung biopsies (SLB), with reports that it has a higher diagnostic yield since anatomical structures remain intact (49). However, since these samples are taken 

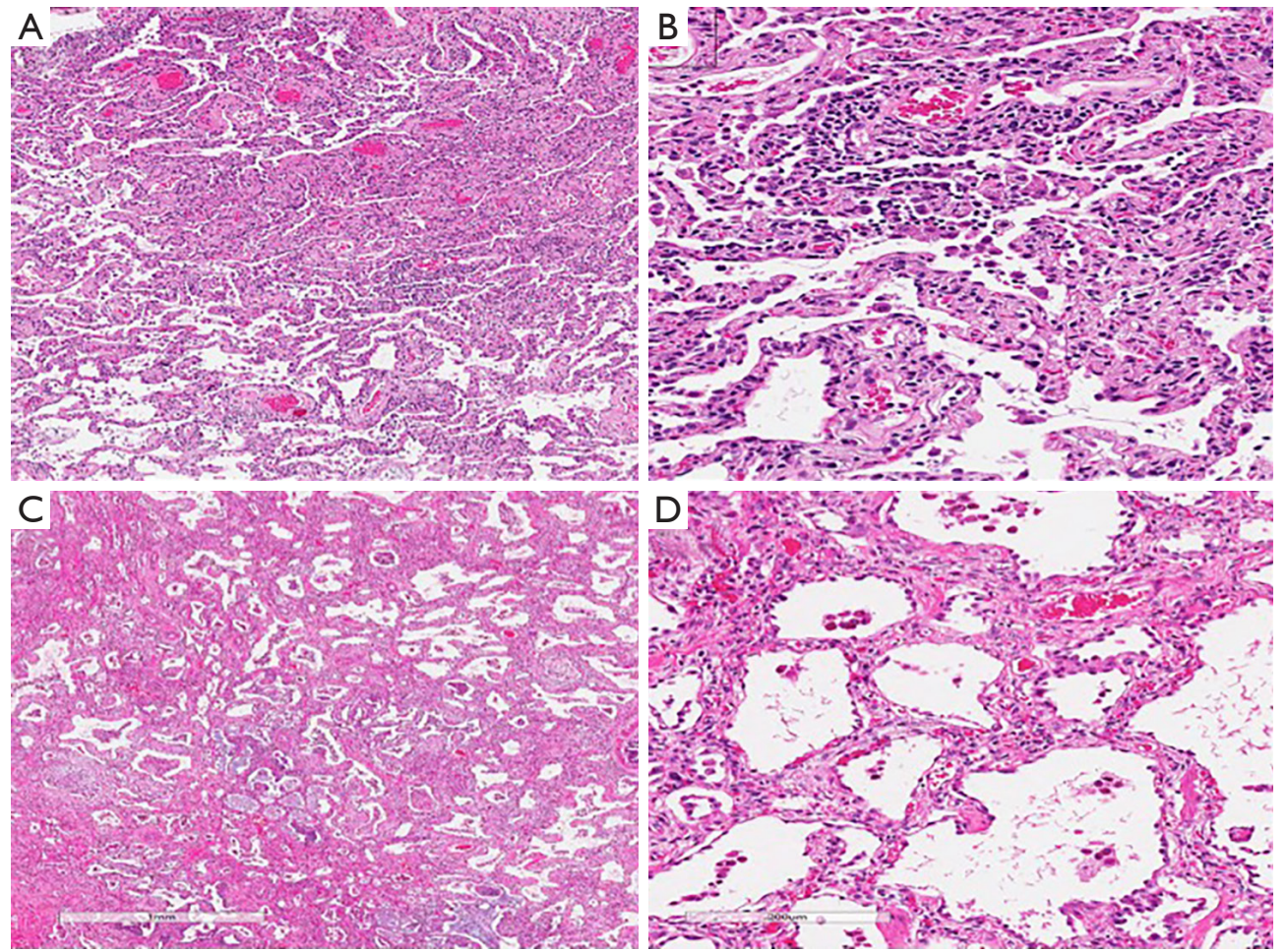

Figure 4 NSIP features seen on a surgical lung biopsy of a patient with AS. (A,B) Explant pneumonectomy from a woman in the 5 th decade of life with AS, positive for anti-PL12. The patient developed acute hypoxic respiratory failure despite aggressive immunosuppression. (A) Diffuse interstitial thickening without architectural distortion or honeycomb change, consistent with NSIP (hematoxylin-eosin, original magnification $\times 20$ ). (B) Higher magnification showing the thickened alveolar septa (arrows indicate fibroblast plugs) (hematoxylin-eosin, original magnification $\times 100)$. (C,D) A woman in her 7th decade of life with AS, positive for anti-PL12. Surgical lung biopsy showing (C) mild, diffuse interstitial thickening consistent with NSIP (hematoxylin-eosin, original magnification $\times 20$ ) and (D) the interstitial infiltrate is predominantly cellular rather than fibrotic. It is composed mainly of lymphocytes (hematoxylin-eosin, original magnification $\times 100$ ).

from a centrilobular location, the peripheral landmarks often required for UIP patterns are absent (49). Thus, when surgical lung biopsy is performed, NSIP is the most common finding (Figure 4); while UIP, organizing pneumonia, or diffuse alveolar damage are observed in less than $20 \%$ of patients (50). The combination of NSIP and organizing pneumonia is more common than either in isolation (12).

In a retrospective study of 20 lung biopsies in patients with anti-Jo- 1 and anti-PL-7 antibodies, of the 20 patients with anti-Jo-1 antibody, $50 \%$ had diffuse alveolar damage and $35 \%$ had usual interstitial pneumonia (UIP) on surgical lung biopsy (51). Another retrospective analysis of the histology of 22 patients with anti-Jo-1 antibody demonstrated $45 \%$ with UIP, $55 \%$ with DAD and $14 \%$ with NSIP, which contradicts prior reports suggesting that NSIP is the most common histopathologic diagnosis in myositis- associated ILD (46). It is plausible that the histopathologic findings in these patients differed from prior reports since a large proportion of patient specimens were from those with severe disease including those undergoing lung transplant or postmortem analysis. More importantly, survival analysis in this biopsy cohort demonstrates that DAD portends a poor prognosis, with a significant 7.4-year reduction in median survival compared to patients without histopathologic or clinical evidence of DAD (46).

It can therefore be concluded that the spectrum of pathologic changes found in AS is similar to those seen in an "idiopathic" setting or in the setting of other conditions associated with organizing pneumonia, diffuse alveolar damage, DAD or UIP. Similarly, as in other settings, the prognosis for organizing pneumonia and NSIP is better than the prognosis for diffuse alveolar damage or UIP. To date, no pathologic findings have been shown to be specific 
for AS. Given the non-specific nature of the pathologic findings and the fact that this entity is defined on the basis of clinical features and serology, the utility of surgical lung biopsies in this setting is questionable. It is likely that lung biopsies are performed in patients in whom the possibility of AS has not yet been entertained, or in whom a competing alternative diagnosis (such as infection) is thought to be high. The precise timing of an autoimmune work-up in the evaluation of such patients remains open to discussion, but it seems clear that early evaluation for autoimmunity (including anti-synthetase antibodies) has the potential to reduce unnecessary tissue sampling. Further, for those in whom a diagnosis of idiopathic interstitial pneumonia is entertained, clinicians should consider screening periodically for development of symptoms/signs of AS, and if suspected, autoantibodies should be checked on an ad hoc basis. It is also paramount that the ILD pulmonologist be familiar with the antibody profile available in the local laboratory and consider send-outs for extended profile if AS-ILD is strongly suspected. Such a vigorous approach is key to obviate unnecessary procedures and institute appropriate treatment, as early as possible. An example of organizing pneumonia in a case of AS is shown in Figure 3. The adjacent interstitium is diffusely thickened. Whether this latter finding represents NSIP or the expected degree of interstitial thickening in organizing pneumonia is often a matter of judgement.

\section{Diagnosis of ILD and resultant complications in AS}

ILD is a recognized manifestation of AS, identified in $75-89 \%$ of cases $(9,52,53)$ and can often be the sole manifestation of the disease. Although ILD commonly presents with a sudden or gradual onset of exertional dyspnea and difficult-to-control dry cough, other clinical symptoms include chest pain, physical exercise intolerance, dyspnea at rest or even acute respiratory failure (1). Pulmonary function tests (PFT) reveal a typical restrictive pattern with reduction in the diffusing capacity of carbon monoxide (44). Total lung capacity varies according to the severity of the disease. Serial PFTs are also useful for disease monitoring, in particular, FVC impairment is more routinely followed. FVC reduction out of proportion to lung parenchymal abnormalities may indicate concomitant muscular/ diaphragmatic weakness in those with myositis.

Additional lab work includes measurement of creatinine kinase (CK) and lactate dehydrogenase (LDH) however studies have shown these are typically lower in patients with little to no muscle involvement (23). Several serum biomarkers have been studied to aid in diagnosis and monitoring disease activity of ILD. Among these, Krebs von den lungen 6 (KL-6), a mucin-like glycoprotein whose overexpression in regenerating type II epithelial cells is related to the presence of fibrotic lung disease, has generated particular interest since KL-6 and pulmonary surfactant protein D (SP-D) levels are associated with the activity and severity of ILD (23). Additionally, the combination of CXCL10, MMP-7, and IL-12 has been found to help distinguish anti-Jo-1 antibody associated ILD from idiopathic pulmonary fibrosis with $100 \%$ sensitivity and $100 \%$ specificity (46). Although current data is promising, the use of serum biomarkers is not yet recommended or available for routine clinical use.

A retrospective analysis looking for pulmonary hypertension in 203 patients with AS postulated that echocardiogram should be performed, particularly in the presence of unexplained or severe dyspnea. It was highlighted that since sera from patients with anti-Jo1 antibody-positive AS can activate endothelial cells in vitro (11), the diagnosis of pulmonary hypertension is of particular importance not only in specific management but also patient outcome. Of the $45 \%$ of patients screened with transthoracic echocardiography, there was a $7.9 \%$ prevalence of pulmonary hypertension (of whom $81.3 \%$ were severe) based on right-heart catheterization. Although this value was felt to underestimate the prevalence, pulmonary hypertension was significantly associated with a lower survival rate with a 3 -year survival rate of $58 \%$ (11). Thus, we recommend a low threshold for obtaining transthoracic echocardiogram in patients with AS-ILD.

\section{Treatment}

There is no standardized approach to treatment of AS-ILD due to absence of randomized controlled trials comparing various agents specifically for this disorder. Therefore, choice of immunosuppression typically follows treatment strategies adopted for ILD secondary to inflammatory myopathies in general. A treatment algorithm (Figure 5) may be considered.

Since the condition is extremely rare, the authors reiterate that the suggestions for treatment recommendation are purely based on clinical experience with similar conditions and the decision to treat is based on assessment of disease severity by combination of symptom surveys, pulmonary function testing and HRCT-quantified disease. Due to 


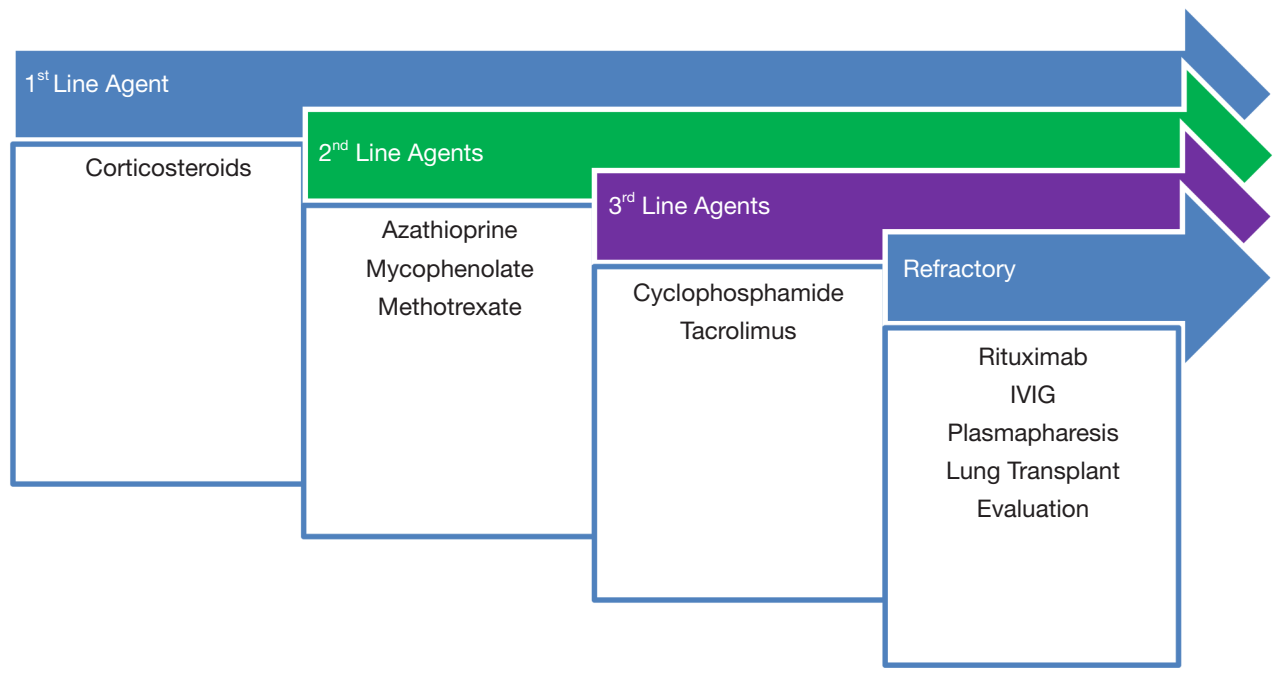

Figure 5 Treatment Algorithm for AS. First-line therapy involves corticosteroids. Second-line agents are azathioprine, mycophenolate, tacrolimus. Third-line agents, or rescue therapy include cyclophosphamide and rituximab. Cyclophosphamide can be considered first line for severe/rapidly declining respiratory failure.

multi-system nature of disease, often a rheumatologist familiar with ILD is part of critical decision-making process.

Corticosteroids are the cornerstone of immunosuppressive therapy due to early studies involving DM/PM-associated ILD that demonstrated a $30-40 \%$ improvement of subjects treated with prednisone and $20-40 \%$ stabilized based on symptoms and pulmonary function (54). Due to the lack of randomized control trials, there are no current evidence-based medicine guidelines outlining a recommended treatment strategy. Based on our clinical practice, we recommend initiating corticosteroid therapy starting oral Prednisone at $1 \mathrm{mg} / \mathrm{kg} /$ day and monitoring for improvement in pulmonary \& myositis symptoms. In patients experiencing acute respiratory failure and rapid decline, higher doses of methylprednisolone (approximating $7.5 \mathrm{mg} / \mathrm{kg}$ for 3 days) may be warranted. There is no specific method to tapering steroid dosing but in our clinical practice, we adopt a gradual taper over 6-8 weeks to a maintenance dose. It is not unusual to increase the dose to the previous effective dose if the patient experiences relapse of symptoms during treatment. We recommend monitoring spirometry every 3-6 months and annual chest imaging by HRCT. It is important to note that controlled trials have not been performed to establish the superiority of corticosteroids compared to other immunosuppressive agents in the initial management of active disease (3).
Given the extended duration of corticosteroid treatment and concern for corticosteroid resistance (55), we often initiate other immunosuppressive medications, such as mycophenolate mofetil or azathioprine. These agents are typically initiated at a lower dose and gradually increased to maximum tolerable dose over a few weeks. Corticosteroidsparing agents are also useful to induce and maintain remission (4). One particular study followed 11 patients with anti-EJ associated ILD for a median of 46 months and of the five relapsed patients, four were on prednisone monotherapy (56). Patients with anti-EJ associate ILD had high risk of disease progression and those with relapse had NSIP and were on corticosteroid monotherapy, whereas the rate of relapse of ILD was lower in patients on combination therapy. Therefore, prednisone monotherapy is typically not recommended. Further, prolonged corticosteroid monotherapy is associated with both short-term and longterm adverse effects.

Cyclophosphamide is typically reserved only for severe cases of ILD. Its efficacy was demonstrated in a 2015 systematic review analyzed 12 studies involving 319 patients, of whom 141 with IIM-ILD were treated with cyclophosphamide (57). Although both the dosage of cyclophosphamide and the co-administered immunosuppressants varied, approximately $71 \%$ demonstrated improvement in vital capacity or FVC and $69 \%$ had improved DLCO (57). 
There has been growing enthusiasm for the use of rituximab in the treatment of AS. Studies suggest that CD20 depletion therapy might be valuable in the treatment of refractory AS (51). In a series of 8 patients in whom rituximab was used as "rescue therapy" for severe ILD, 7 out of 8 patients had a favorable response measured by improved DLCO and FVC (58). Another retrospective study in 2018 evaluated 25 patients with AS and ILD who were treated with Rituximab for recurrent and or progressive ILD despite being on immunosuppressant therapy. Pulmonary variables including CT severity score, forced vital capacity, total lung capacity was measured preand post-RTX. At 12 months, the CT score and forced vital capacity were noted to be either stable or improved in $88 \%$ and $79 \%$ of subjects, and the total lung capacity (\%) was increased from $56 \pm 13$ to $64 \pm 13$ (59). DLCO (\%) was measured over the course of 3 years and increased from $42 \pm 17$ to $70 \pm 20(59)$.

Early studies showed that corticosteroid dose reduction in patients anti-ARS associated ILD was associated with relapse, and combination therapy of corticosteroids and tacrolimus was effective in improving FVC, DLCO and decreasing the number of relapses $(12,60,61)$. Additionally, Tacrolimus may have higher greater potency and an improved safety profile when compared to cyclosporine (62). However, tacrolimus is often used as a third line agent due to high risk for systemic toxicity and medication interactions.

The use of intravenous immunoglobulin (IVIG) in the treatment of AS and ILD has been evaluated in a retrospective case series published in 2019 demonstrated that IVIG stabilized the lung function, increased the mean FVC and DLCO in patients with ILD (63). Although it also decreased the mean prednisone dose over time in patients with AS, roughly $53 \%$ of patients experienced side effects. Similarly, rare case reports have discussed the use of therapeutic plasma exchange for rapidly progressive ILD, refractory to treatment and have demonstrated favorable results with improvement of myositis and respiratory status with decreasing antibody index $(64,65)$.

These aforementioned medications take various routes to suppress normal immune function in an effort to combat aberrant pathogenic autoimmunity. Prescribing providers should be familiar with their indications as well as their complications (Table 3) with regular monitoring for organ damage, increased risk of opportunistic infections, and malignancy (particularly lymphoma and skin cancer). We would like to emphasize the importance of antibiotic prophylaxis (Trimethoprim/Sulfamethoxazole, Dapsone, Pentamidine) due to the risk of opportunistic infections while on long-term steroid therapy. Treatment decisions should be made keeping patient preference, comorbidities, physician experience with medications and overall goals of care in mind.

Other treatment strategies involve the addition of supplemental oxygen since these patients often develop hypoxia with progression of their lung disease. Referral to pulmonary rehabilitation is also recommended since studies have shown a clear benefit from exercise training in patients with ILD, regardless of etiology (66).

Lung transplant should also be considered for patients with advanced ILD whose clinical status has progressively declined despite maximal medical therapy. Although data on patients with AS receiving lung transplants is limited, studies have shown that patients with $\mathrm{PM} / \mathrm{DM}$ undergoing double lung transplant have similar 1- and 5-year survival rates to IPF recipients (67). The international Society of Heart and Lung Transplantation (ISHLT) have developed specific guidelines to identify eligible candidates (Table 4). Additionally, close attention should be given to comorbidities associated with AS. Gastroesophageal reflux disease (GERD) is commonly observed in patients with ILD and is a known risk factor for both aspiration and microaspiration. In these patients, diligent evaluation of the upper gastrointestinal tract needs to be performed as it can preclude lung transplantation at some centers. Patient should be evaluated for reflux and dysphagia symptoms. Any symptoms suggesting reflux or dysphagia warrant further investigations with barium esophagogram, 24-hour pH probe with esophageal manometry, esophagogastroduodenoscopy, and gastric emptying study. Lung transplantation can be considered for properly selected candidates with worsening ILD due to AS despite a trial of immunosuppressive therapy. Current guidelines from the American Thoracic Society (ATS) recommend the addition of antacid treatments to decrease the risk for microaspiration-associated lung injury or damage (69).

Other important co-morbidities to consider include both pulmonary hypertension and a discussion on whether to screen for malignancy. The association between dermatomyositis and malignancy has long been proposed as a paraneoplastic phenomenon, similarly several case series and reports have suggested that AS may also be associated with the presence of malignancies (70). Despite their small sample size of patients some authors have proposed there is increased risk of malignancy in patients within 6-12 months of 
Table 3 Treatment strategies for AS specific ILD

\begin{tabular}{|c|c|c|c|c|}
\hline Medications & Suggested dose & Baseline testing & Test frequency & Major adverse effects \\
\hline Corticosteroids & $\begin{array}{l}\text { Prednisone: } 1 \mathrm{mg} / \mathrm{kg} \text { daily } \\
\text { Upper limit: } 60-80 \mathrm{mg} \text { daily }\end{array}$ & $\begin{array}{l}\text { Bone density } \\
\text { Hemoglobin A1c }\end{array}$ & $\begin{array}{l}\text { After } 1 \text { year, then every } 2-3 \text { years } \\
\text { if stable or more frequently if bone } \\
\text { density decreased }\end{array}$ & $\begin{array}{l}\text { Hyperglycemia } \\
\text { Hypertension } \\
\text { Osteoporosis } \\
\text { Cataracts } \\
\text { Sleep disturbances }\end{array}$ \\
\hline Azathioprine & $1.0-2.0 \mathrm{mg} / \mathrm{kg} /$ day & $\begin{array}{l}\text { CBC, CMP, thiopurine } \\
\text { S-methyltransferase } \\
\text { (TPMT), pregnancy }\end{array}$ & Monthly, then every 3 months & $\begin{array}{l}\text { BM suppression } \\
\text { Hepatotoxicity, Pancreatitis }\end{array}$ \\
\hline Mycophenolate & $500-1,500$ mg PO BID & CBC, CMP, pregnancy & Monthly, then every 3 months & $\begin{array}{l}\text { BM suppression } \\
\text { GI symptoms (e.g., diarrhea) } \\
\text { Increase miscarriage \& } \\
\text { congenital abnormalities }\end{array}$ \\
\hline Tacrolimus & $\begin{array}{l}1 \mathrm{mg} \text { twice daily (target trough } \\
\text { levels } 5-8 \mathrm{ng} / \mathrm{mL} \text { ) }\end{array}$ & CBC, CMP, pregnancy test & Monthly & $\begin{array}{l}\text { CKD } \\
\text { Hypertension } \\
\text { Tremors }\end{array}$ \\
\hline Cyclophosphamide & IV CYC monthly $\times 6$ months & $\begin{array}{l}\text { CBC, CMP, pregnancy, } \\
\text { hepatitis panel, T-spot, } \\
\text { urinalysis, age-appropriate } \\
\text { cancer screening }\end{array}$ & $\begin{array}{l}\text { Monthly } \times 1 \text { year, then every } 3 \\
\text { months }\end{array}$ & $\begin{array}{l}\text { Malignancy, Cytopenias, } \\
\text { Hemorrhagic cystitis, Sterility }\end{array}$ \\
\hline Rituximab & $\begin{array}{l}1 \mathrm{gm} \times 2 \text { doses, } 2 \text { weeks } \\
\text { apart }\end{array}$ & $\begin{array}{l}\text { CBC, CMP, HIV, hepatitis } \\
\text { panel, T-spot test }\end{array}$ & Prior to each infusion & $\begin{array}{l}\text { Infection, Neutropenia, Infusion } \\
\text { reaction }\end{array}$ \\
\hline
\end{tabular}

AS, anti-synthetase syndrome; ILD, interstitial lung disease.

Table 4 Lung transplantation referral indications and listing for ILD (68)

Referral:

- Histopathologic or radiographic evidence of usual interstitial pneumonitis (UIP) or fibrosing non-specific interstitial pneumonitis (NSIP), regardless of lung function

- Abnormal lung function: FVC $<80 \%$ predicted or diffusion capacity of the lung for carbon monoxide $\left(\mathrm{D}_{\text {LCO }}\right)<40 \%$ predicted

- Any dyspnea or functional limitation attributable to lung disease

- Any oxygen requirement, even if only during exertion

Timing of listing:

- Decline in FVC $>10 \%$ during 6-month follow-up

- Decline in $\mathrm{D}_{\mathrm{LCO}}>15 \%$ during 6-month follow-up

- Desaturation $<88 \%$ or distance $<250 \mathrm{~m}$ on $6 \mathrm{MWT}$ or $>50$ m decline on 6MWD over 6 months

- Pulmonary hypertension

- Hospitalization due to respiratory decline, pneumothorax, or acute exacerbations

Specific for AS \& other inflammatory lung diseases referral for lung transplantation is prudent if despite a clinically indicated trial of medical therapy any of the following are present:

- Dyspnea or functional limitation

- An oxygen requirement

- Declining lung function

ILD, interstitial lung disease; FVC, forced vital capacity. 
diagnosis with AS (71), with prevalence rates up to $14 \%$ (72). However, a larger retrospective analysis of 233 patients with AS found that the frequency of malignancy was only $1.7 \%$ within three years from diagnosis, not more than the general population in France (71). Therefore, there is no evidence to support cancer screening outside of the recommended guidelines.

\section{Conclusions}

AS is an autoimmune disorder characterized by autoantibodies to specific aminoacyl-transfer RNA synthetases resulting in clinical manifestations such as myositis, Raynaud phenomenon, non-erosive arthritis, fever, and mechanic's hands. Although each autoantibody has a distinct phenotype, the disease itself is associated with a high burden of ILD that is often more severe and rapidly progressive when compared to other IIM. Lung biopsies should be infrequently utilized since diagnosis is typically made based on HRCT findings, serologic data, pulmonary function testing, physical examination, and patient symptoms. Common radiographic findings include an interstitial pattern or ground glass lesions, and patients with organizing pneumonia usually have a more favorable prognosis. Treatment options include corticosteroids and immunosuppressants, with Rituximab often reserved as salvage therapy in refractory cases. Due to research being limited primarily to retrospective studies and case reports, data is severely lacking on each agents' efficacy based on phenotype. We recommend further research in the form of prospective studies to aid physicians when making treatment choices in order to improve the chance of patient survival.

\section{Acknowledgments}

Funding: None.

\section{Footnote}

Reporting Checklist: The authors have completed the Narrative Review reporting checklist. Available at https:// dx.doi.org/10.21037/jtd-20-3328

Peer Review File: Available at https://dx.doi.org/10.21037/ jtd-20-3328

Conflicts of Interest: All authors have completed the ICMJE uniform disclosure form (available at https://dx.doi. org/10.21037/jtd-20-3328). The authors have no conflicts of interest to declare.

Ethical Statement: The authors are accountable for all aspects of the work in ensuring that questions related to the accuracy or integrity of any part of the work are appropriately investigated and resolved.

Open Access Statement: This is an Open Access article distributed in accordance with the Creative Commons Attribution-NonCommercial-NoDerivs 4.0 International License (CC BY-NC-ND 4.0), which permits the noncommercial replication and distribution of the article with the strict proviso that no changes or edits are made and the original work is properly cited (including links to both the formal publication through the relevant DOI and the license). See: https://creativecommons.org/licenses/by-nc-nd/4.0/.

\section{References}

1. Antisynthetase syndrome. Orphanet Encylopedia. Available online: http://www.orpha.net/data/patho/GB/ukantisynthetase.pdf2001

2. Inflammatory Myopathies Fact Sheet: National Institute of Neurological Disorders and Stroke U.S. Department of Health and Human Services; 2019. Available online: https://www.ninds.nih.gov/disorders/patient-caregivereducation/fact-sheets/inflammatory-myopathies-fact-sheet

3. Witt LJ, Curran JJ, Strek ME. The Diagnosis and Treatment of Antisynthetase Syndrome. Clin Pulm Med 2016;23:218-26.

4. Douglas WW, Tazelaar HD, Hartman TE, et al. Polymyositis-dermatomyositis-associated interstitial lung disease. Am J Respir Crit Care Med 2001;164:1182-5.

5. Betteridge Z, Gunawardena H, North J, et al. Antisynthetase syndrome: a new autoantibody to phenylalanyl transfer RNA synthetase (anti-Zo) associated with polymyositis and interstitial pneumonia. Rheumatology (Oxford) 2007;46:1005-8.

6. Mammen AL. Dermatomyositis and polymyositis: Clinical presentation, autoantibodies, and pathogenesis. Ann N Y Acad Sci 2010;1184:134-53.

7. Kalluri M, Oddis CV. Pulmonary manifestations of the idiopathic inflammatory myopathies. Clin Chest Med 2010;31:501-12.

8. Walker EJ, Tymms KE, Webb J, et al. Improved detection of anti-Jo-1 antibody, a marker for myositis, using purified histidyl-tRNA synthetase. J Immunol Methods 
1987;96:149-56.

9. Bernstein RM, Morgan SH, Chapman J, et al. Anti-Jo-1 antibody: a marker for myositis with interstitial lung disease. Br Med J (Clin Res Ed) 1984;289:151-2.

10. Maeda MH. Idiopathic Inflammatory Myopathy and Anti-aminoacyl-tRNA Synthetase Antibody. Brain Nerve 2018;70:439-48.

11. Hervier B, Meyer A, Dieval C, et al. Pulmonary hypertension in antisynthetase syndrome: prevalence, aetiology and survival. Eur Respir J 2013;42:1271-82.

12. Solomon J, Swigris JJ, Brown KK. Myositis-related interstitial lung disease and antisynthetase syndrome. J Bras Pneumol 2011;37:100-9.

13. Chatterjee S, Prayson R, Farver C. Antisynthetase syndrome: not just an inflammatory myopathy. Cleve Clin J Med 2013;80:655-66.

14. Watanabe K, Handa T, Tanizawa K, et al. Detection of antisynthetase syndrome in patients with idiopathic interstitial pneumonias. Respir Med 2011;105:1238-47.

15. Love LA, Leff RL, Fraser DD, et al. A new approach to the classification of idiopathic inflammatory myopathy: myositis-specific autoantibodies define useful homogeneous patient groups. Medicine (Baltimore) 1991;70:360-74.

16. Connors GR, Christopher-Stine L, Oddis CV, et al. Interstitial lung disease associated with the idiopathic inflammatory myopathies: what progress has been made in the past 35 years? Chest 2010;138:1464-74.

17. Bohan A, Peter JB. Polymyositis and dermatomyositis (first of two parts). N Engl J Med 1975;292:344-7.

18. Greco M, Garcia de Yebenes MJ, Alarcon I, et al. Antisynthetase syndrome: Clinical value of Solomon's and Connors' diagnosis criteria. Ann Rheum Dis 2019;78:1789.

19. Cavagna L, Trallero-Araguás E, Meloni F, et al. Influence of Antisynthetase Antibodies Specificities on Antisynthetase Syndrome Clinical Spectrum Time Course. J Clin Med 2019;8:2013.

20. Wernham M, Montague SJ. Mechanic's hands and hiker's feet in antisynthetase syndrome. CMAJ 2017;189:E1365.

21. Kalluri M, Sahn SA, Oddis CV, et al. Clinical profile of anti-PL-12 autoantibody. Cohort study and review of the literature. Chest 2009;135:1550-6.

22. Disorders National Organization for Rare Disorders. Danbury, CT: Antisynthetase Syndrome, 2018.

23. Hamaguchi Y, Fujimoto M, Matsushita T, et al. Common and distinct clinical features in adult patients with antiaminoacyl-tRNA synthetase antibodies: heterogeneity within the syndrome. PLoS One 2013;8:e60442.
24. Trallero-Araguás E, Grau-Junyent JM, Labirua-Iturburu A, et al. Clinical manifestations and long-term outcome of anti-Jo1 antisynthetase patients in a large cohort of Spanish patients from the GEAS-IIM group. Semin Arthritis Rheum 2016;46:225-31.

25. Labirua-Iturburu A, Selva-O'Callaghan A, Vincze M, et al. Anti-PL-7 (anti-threonyl-tRNA synthetase) antisynthetase syndrome: clinical manifestations in a series of patients from a European multicenter study (EUMYONET) and review of the literature. Medicine (Baltimore) 2012;91:206-11.

26. Marguerie C, Bunn CC, Beynon HL, et al. Polymyositis, pulmonary fibrosis and autoantibodies to aminoacyl-tRNA synthetase enzymes. Q J Med 1990;77:1019-38.

27. Marie I, Josse S, Hatron PY, et al. Interstitial lung disease in anti-Jo-1 patients with antisynthetase syndrome. Arthritis Care Res (Hoboken) 2013;65:800-8.

28. Hirakata M. Autoantibodies to aminoacyl-tRNA synthetases. Intern Med 2005;44:527-8.

29. Mirrakhimov A. Antisynthetase syndrome: a review of etiopathogenesis, diagnosis and management. Curr Med Chem 2015;22:1963-75.

30. Stone KB, Oddis CV, Fertig N, et al. Anti-Jo-1 antibody levels correlate with disease activity in idiopathic inflammatory myopathy. Arthritis Rheum 2007;56:3125-31.

31. Andersson H, Sem M, Lund MB, et al. Long-term experience with rituximab in anti-synthetase syndromerelated interstitial lung disease. Rheumatology (Oxford) 2015;54:1420-8.

32. Nishikai M, Ohya K, Kosaka M, et al. Anti-Jo-1 antibodies in polymyositis or dermatomyositis: evaluation by ELISA using recombinant fusion protein Jo-1 as antigen. Br J Rheumatol 1998;37:357-61.

33. Targoff IN, Reichlin M. Measurement of antibody to Jo-1 by ELISA and comparison to enzyme inhibitory activity. J Immunol 1987;138:2874-82.

34. Fischer A, Swigris JJ, du Bois RM, et al. Anti-synthetase syndrome in ANA and anti-Jo-1 negative patients presenting with idiopathic interstitial pneumonia. Respir Med 2009;103:1719-24.

35. Aggarwal R, Cassidy E, Fertig N, et al. Patients with nonJo-1 anti-tRNA-synthetase autoantibodies have worse survival than Jo-1 positive patients. Ann Rheum Dis 2014;73:227-32.

36. Lega JC, Fabien N, Reynaud Q, et al. The clinical phenotype associated with myositis-specific and associated autoantibodies: a meta-analysis revisiting the so-called antisynthetase syndrome. Autoimmun Rev 2014;13:883-91.

37. Hervier B, Devilliers H, Stanciu R, et al. Hierarchical 
cluster and survival analyses of antisynthetase syndrome: phenotype and outcome are correlated with antitRNA synthetase antibody specificity. Autoimmun Rev 2012;12:210-7.

38. Marie I, Josse S, Decaux O, et al. Comparison of long-term outcome between anti-Jo1- and anti-PL7/PL12 positive patients with antisynthetase syndrome. Autoimmun Rev 2012;11:739-45.

39. Yoshifuji H, Fujii T, Kobayashi S, et al. Anti-aminoacyltRNA synthetase antibodies in clinical course prediction of interstitial lung disease complicated with idiopathic inflammatory myopathies. Autoimmunity 2006;39:233-41.

40. Chawla A, Kumar T, Mukherjee P. Antisynthetase syndrome: Initial and follow-up imaging features. Lung India 2018;35:523-5.

41. Waseda Y, Johkoh T, Egashira R, et al. Antisynthetase syndrome: Pulmonary computed tomography findings of adult patients with antibodies to aminoacyl-tRNA synthetases. Eur J Radiol 2016;85:1421-6.

42. Debray MP, Borie R, Revel MP, et al. Interstitial lung disease in anti-synthetase syndrome: initial and follow-up CT findings. Eur J Radiol 2015;84:516-23.

43. Mira-Avendano I, Abril A, Burger CD, et al. Interstitial Lung Disease and Other Pulmonary Manifestations in Connective Tissue Diseases. Mayo Clin Proc 2019;94:309-25.

44. Badshah A, Haider I, Pervez S, et al. Antisynthetase syndrome presenting as interstitial lung disease: a case report. J Med Case Rep 2019;13:241.

45. Chung JH, Cox CW, Montner SM, et al. CT Features of the Usual Interstitial Pneumonia Pattern: Differentiating Connective Tissue Disease-Associated Interstitial Lung Disease From Idiopathic Pulmonary Fibrosis. AJR Am J Roentgenol 2018;210:307-13.

46. Richards TJ, Eggebeen A, Gibson K, et al. Characterization and peripheral blood biomarker assessment of anti-Jo-1 antibody-positive interstitial lung disease. Arthritis Rheum 2009;60:2183-92.

47. Furini F, Carnevale A, Casoni GL, et al. The Role of the Multidisciplinary Evaluation of Interstitial Lung Diseases: Systematic Literature Review of the Current Evidence and Future Perspectives. Front Med (Lausanne) 2019;6:246.

48. Tirelli C, Morandi V, Valentini A, et al. Multidisciplinary Approach in the Early Detection of Undiagnosed Connective Tissue Diseases in Patients With Interstitial Lung Disease: A Retrospective Cohort Study. Front Med (Lausanne) 2020;7:11.

49. Troy LK, Hetzel J. Lung cryobiopsy and interstitial lung disease: What is its role in the era of multidisciplinary meetings and antifibrotics? Respirology 2020;25:987-96.

50. Sauty A, Rochat T, Schoch OD, et al. Pulmonary fibrosis with predominant CD8 lymphocytic alveolitis and antiJo-1 antibodies. Eur Respir J 1997;10:2907-12.

51. Yousem SA, Gibson K, Kaminski N, et al. The pulmonary histopathologic manifestations of the anti-Jo-1 tRNA synthetase syndrome. Mod Pathol 2010;23:874-80.

52. Schnabel A, Reuter M, Biederer J, et al. Interstitial lung disease in polymyositis and dermatomyositis: clinical course and response to treatment. Semin Arthritis Rheum 2003;32:273-84.

53. Späth M, Schröder M, Schlotter-Weigel B, et al. The long-term outcome of anti-Jo-1-positive inflammatory myopathies. J Neurol 2004;251:859-64.

54. Frazier AR, Miller RD. Interstitial pneumonitis in association with polymyositis and dermatomyositis. Chest 1974;65:403-7.

55. Fischer A, Brown KK, Du Bois RM, et al. Mycophenolate mofetil improves lung function in connective tissue disease-associated interstitial lung disease. J Rheumatol 2013;40:640-6.

56. Sasano H, Hagiwara E, Kitamura H, et al. Long-term clinical course of anti-glycyl tRNA synthetase (anti-EJ) antibody-related interstitial lung disease pathologically proven by surgical lung biopsy. BMC Pulm Med 2016;16:168.

57. Ge Y, Peng Q, Zhang S, et al. Cyclophosphamide treatment for idiopathic inflammatory myopathies and related interstitial lung disease: a systematic review. Clin Rheumatol 2015;34:99-105.

58. Keir GJ, Maher TM, Hansell DM, et al. Severe interstitial lung disease in connective tissue disease: rituximab as rescue therapy. Eur Respir J 2012;40:641-8.

59. Doyle TJ, Dhillon N, Madan R, et al. Rituximab in the Treatment of Interstitial Lung Disease Associated with Antisynthetase Syndrome: A Multicenter Retrospective Case Review. J Rheumatol 2018;45:841-50.

60. Wilkes MR, Sereika SM, Fertig N, et al. Treatment of antisynthetase-associated interstitial lung disease with tacrolimus. Arthritis Rheum 2005;52:2439-46.

61. Koreeda Y, Higashimoto I, Yamamoto M, et al. Clinical and pathological findings of interstitial lung disease patients with anti-aminoacyl-tRNA synthetase autoantibodies. Intern Med 2010;49:361-9.

62. Klein IH, Abrahams A, van Ede T, et al. Different effects of tacrolimus and cyclosporine on renal hemodynamics and blood pressure in healthy subjects. Transplantation 2002;73:732-6. 
63. Huapaya JA, Hallowell R, Silhan L, et al. Long-term treatment with human immunoglobulin for antisynthetase syndrome-associated interstitial lung disease. Respir Med 2019;154:6-11.

64. Omotoso BA, Ogden MI, Balogun RA. Therapeutic plasma exchange in antisynthetase syndrome with severe interstitial lung disease. J Clin Apher 2015;30:375-9.

65. Ning Y, Yang G, Sun Y, et al. Efficiency of Therapeutic Plasma-Exchange in Acute Interstitial Lung Disease, Associated With Polymyositis/Dermatomyositis Resistant to Glucocorticoids and Immunosuppressive Drugs: A Retrospective Study. Front Med (Lausanne) 2019;6:239.

66. Dowman LM, McDonald CF, Hill CJ, et al. The evidence of benefits of exercise training in interstitial lung disease: a randomised controlled trial. Thorax 2017;72:610-9.

67. Dincheva G, Rajalingam K, Chen JW, et al. Polymyositis and Dermatomyositis in Lung Transplantation: The National U.S. Experience. Am J Respir Crit Care Med 2018;197:A7373.

Cite this article as: Sawal N, Mukhopadhyay S, Rayancha S, Moore A, Garcha P, Kumar A, Kaul V. A narrative review of interstitial lung disease in anti-synthetase syndrome: a clinical approach. J Thorac Dis 2021;13(9):5556-5571. doi: 10.21037/jtd$20-3328$
68. Weill D, Benden C, Corris PA, et al. A consensus document for the selection of lung transplant candidates: 2014--an update from the Pulmonary Transplantation Council of the International Society for Heart and Lung Transplantation. J Heart Lung Transplant 2015;34:1-15.

69. Raghu G, Rochwerg B, Zhang Y, et al. An Official ATS/ ERS/JRS/ALAT Clinical Practice Guideline: Treatment of Idiopathic Pulmonary Fibrosis. An Update of the 2011 Clinical Practice Guideline. Am J Respir Crit Care Med 2015;192:e3-19.

70. Legault D, McDermott J, Crous-Tsanaclis AM, et al. Cancer-associated myositis in the presence of antiJo1 autoantibodies and the antisynthetase syndrome. J Rheumatol 2008;35:169-71.

71. Cojocaru M, Cojocaru IM, Chicos B. New Insights into Antisynthetase Syndrome. Maedica (Bucur) 2016;11:130-5.

72. Castañeda-Pomeda M, Prieto-González S, Grau JM. Antisynthetase syndrome and malignancy: our experience. J Clin Rheumatol 2011;17:458. 